\title{
UNIFICAZIONE E ARMONIZZAZIONE DEL DIRITTO SOCIETARIO IN EUROPA
}

\author{
Diego Corapi \\ Professore ordinario nell'Università di Roma-Tor Vergata
}

Resumo:

A finalidade dos Tratados de Roma e Maastricht é a iniciação de um mercado único europeu, a qual se exprime nas liberdades de circulação de mercadoria, de capitais, de pessoas e de estabelecimento das empresas.

$\mathrm{O}$ ordenamento comunitário, com seus regulamentos, visa abater as barreiras entre os diversos países membros através da realização de uma equivalência substancial entre seus sistemas normativos.

O objetivo não é a "unificação", tampouco a "uniformização" das regras nacionais, mas sim a "harmonização" À harmonização não interessa que as regras sejam formalmente idênticas, mas que sua adoção importe em resultados substancialmente correspondentes, impondo-se a cada Estadomembro a adoção das medidas que, no contexto de cada um dos seus ordenamentos, resultem as mais adequadas.

A harmonização da disciplina das sociedades é essencial para tornar neutra as escolhas de sua localização no âmbito do mercado único europeu.

A matéria societária foi também objeto de um projeto de disciplina comunitária, o qual visa realizar uma regulamentação unitária e igual em cada um dos ordenamentos jurídicos dos países membos, a fim de oferecer um instrumento às empresas que operam transnacionalmente.

Iniciativa de mesma envergadura se fez no campo dos institutos de índole contratual que propriciam formas de colaboração entre operadores econômicos (ex.: "joint ventures").

Abstract:

Roma and Maastricht's Treaty's purpose consist on the initiation of an european trading centre, which is expressed by free circulation of commodities, capital, people and enterprises' establishment.

The communitary commandment, with its rules, aim at throwing down the barriers among the different member countries through the realization of a substantial equivalence on their normative system.

The purpose is not "unification", nor "uniformization" of national rules, but "harmonization" To harmonization it doesn't matter if the rules are not the very same, but it's adoption should bring substantial correspondent results, imposing to each State member the adoption of measures that, in their respectives commandments, result the most appropriate. 
The harmonization of societies' discipline is essential due rendering neutral the choices on their position at the scope of the european trading centre.

The societarian matter was also object of a communitary discipline project, which aims the realization of a unitary settlement, equal for every juridical member countries' commandment, in order to offer means to the enterprises which operate transnationaly.

A similar introductory step was take on the field of the institutes with contractual character that appease forms of collaboration among economic operators (ex.: joint ventures).

Unitermos: circulação de mercadoria; mercado único europeu.

1. Il superamento delle barriere nazionali per la creazione di un mercato unico ovvero, in senso ancora più ampio, di uno spazio o unico europeo è la finalità del Trattato di Roma istitutivo della Comunità economica europea e delle successive sue modifiche e integrazioni con il Trattato di Maastricht.

Questa finalità si esprime nella affermazione delle così dette quattro libertà: libertà di circolazione delle merci, dei capitali, delle persone e libertà di stabilimento delle imprese. L'ordinamento comunitario con i suoi regolamenti, che costituiscono fonti sovrardinate alle fonti normative di ognuno dei paesi membri, ha attuato e sta attuando le quattro libertà: i regolamenti abbatono le barriere tra $\mathrm{i}$ diversi paesi membri.

La presenza di normative diverse negli ordinamenti giuridici dei diversi paesi membri, tuttavia, costituisce un elemento di distorsione nell'esercizio delle libertà fondamentali. Il flusso di merci, capitali, persone, lo stabilimento delle imprese in uno o nell'altro dei paesi membri può essere influenzato da fattori non economici ma istituzionali: la presenza di normative diverse, più o meno favorevoli, in materia fiscale, societaria e in generale nella disciplina dell'attività economica è uno degli elementi che condizionano le scelte economiche degli imprenditori.

Di qui l'ulteriore esigenza che l'ordinamento comunitario mira a soddisfare: quella di realizzare una sostanziale equivalenza tra le normative dei diversi paesi membri, in modo da neutralizzare il pericolo che le libere iniziative nel mercato unico siano condizionate da fattori istituzionali diversi. 
2. Particolare è la tecnica giuridica utilizzata per soddisfare la suddetta esigenza.

L'obiettivo non è quello della unificazione delle regole dei diversi ordinamenti giuridici statali (come, invece, in certi settori del commercio oggetto di trattati internazionali: ad es. la Convenzione di Vienna sulla vendita internazionale). Si è ritenuta eccessiva e, forse, non congruente l'imposizione di una normativa identica in tutti gli ordinamenti giuridici considerati. Si è considerato, inverso, che la normativa unica non sarebbe sufficiente in quanto lascerebbe intatte le differenze nella sua interpretazione e applicazione all'interno di ciascun ordinamento.

L'obiettivo non è neppure quello della uniformazione delle regole nazionali (come, invece, nella Convenzione di Roma sulla legge applicabile alle obbligazioni contrattuali). Questa tecnica, invero, consente il superamento di situazioni di conflitto di leggi in quanto impone l'utilizzazione di identici criteri di collegamento. Ma non porta in nessun modo a una coincidenza di contenuto delle leggi materiali applicabili in forza dell'uguale criterio di collegamento.

L'obiettivo è quello - assai originale e ambizioso dell'armonizzazione ovvero avvicinamento delle regole presenti negli ordinamenti dei diversi paesi membri: così l'art. 3, lett. b) e gli artt. 100-102 del Trattato istitutivo della CE.

L'armonizzazione, pur mirando ad un risultato formalmente meno rilevante della vera e propria unificazione normativa, è in realtà più ambiziosa di questa, perchè non si limita ad un risultato formale, ma tende a raggiungere un risultato sostanziale. Non interessa che le regole da applicare siano formalmente identiche, interessa che la loro applicazione porti a risultati sostanzialmente corrispondenti, non contrastanti ma armonici.

Lo strumento per l'attuazione di questo obiettivo è la direttiva (art. 189 del Trattato istitutivo della C.E.) che si rivolge ai singoli Stati membri per imporre l'adozione di quelle misure che nel contesto di ognuno dei loro ordinamenti risultino le più adeguate.

L'art. 177 del Trattato istitutivo dela C.E. assicura poi lo strumento giudiziale (il ricorso alla Corte di giustizia C.E.) per l'armonia nell'interpretazione e applicazione nei diversi Stati membri dei provvedimenti derivanti dall'ordinamento comunitario. 
3. L'art. 54, paragrafo 3, lett. g) del Trattato istitutivo della C.E. prevede il coordinamento delle legislazioni degli Stati membri in materia societaria.

La disciplina delle società, come forme organizzative dell'impresa, rappresenta invero uno dei capitoli più importanti del diritto commerciale e la sua armonizzazione è essenziale per rendere neutre le scelte della loro localizzazione nell'ambito del mercato único europeo.

In attuazione di questo principio, sono state emanate numerose direttive, che gli Stati membri hanno già recepito o hanno in corso di recepimento, su diversi aspetti del diritto delle società (costituzione, aumento di capitale, poteri degli amministratori, bilancio, fusione, etc.).

Per altre parti del diritto delle società sono allo studio diversi progetti o avant projets di direttiva.

Per il quadro dello stato attuale delle direttive comunitarie in materia societaria si veda la Appendice I.

4. La materia societaria è stata oggetto anche di un progetto di disciplina comunitaria, che si propone un diverso obiettivo: quello della istituzione, com uno strumento di vero e proprio diritto comunitario, di un tipo "società europea"

Il progetto mira a realizzare una regolamentazione unitaria e uguale in ognuno degli ordinamenti giuridici dei Paesi membri, per offrire uno strumento a quelle imprese di più vasto respiro che si collocano a livello transnazionale.

L'iniziativa si è tradotta in un progetto di Statuto di Società per Azioni europea che dovrebbe essere oggetto di un regolamento comunitario direttamente applicabile in ognuno degli Stati membri.

5. Infine, una iniziativa nella stessa linea di quella della Società per azioni europea, si è realizzata, invece, nel campo degli istituti di carattere contrattuale che attuano quelle forme di collaborazione tra operatori economici (le c.d. "joint ventures" diverse dalle vere e proprie società.

Il regolamento comunitario n. 2137/85 del 25.7.1985 ha disciplinato il GEIE (Gruppo Europeo di interesse economico). 


\section{APPENDICE \\ STATO DI ADATTAMENTO DELLE DIRETTIVE IN MATERIA DI SOCIETÀ (*)}

Prima direttiva del Consiglio del 9 marzo 1968, n. 68/151, intesa a coordinare, per renderle equivalenti, le garanzie che sono richieste, negli Stati membri, alle società a mente dell'art. 58, secondo comma, del trattato per proteggere gli interessi dei soci e dei terzi (in G.U.C.E. n. I 65 del 14.3.1968, p. 9 s.)

BELGIO

Loi modifiant la législation relative aux sociétés commerciales du 6 mars 1973

DANIMARCA

Act no. 503 of 29th November 1972 to amend the Companies Act 1930

Lov om aendring af lov om aktieselskaber

FRANÇA

GERMANIA

GRECIA

IRLANDIA
Ordonnance n. 69-1176 du 20 décembre 1969 modifiant la loi $n^{\circ} 66-537$ du 24 juillet 1966 sur les sociétés commerciales

Décret n. 69-1177 du 24 décembre 1969 modifiant le décret $\mathrm{n}^{\circ}$ 66-236 du 23 mars 1967 sur les sociétés commerciales et le décret $n^{\circ}$ 67-237 du 23 mars 1967 relatif au registre du commerce

Gesetz für Durchführung der Ersten Richtlinie des Rates der Europäischen Gemeinschaften zur Koordinierung des Gesellschaftsrechts vom 15 August 1969

Presidential Decree n. 409/86 revising Law n. 2190/1920 on public companies limited by shares

Presidential Decree n. 419/86 revising Law n. 2190/1920 on "private limited companies" and "sociétés en commandite par actions"

Statutory Instrument n. 163 of 1973European Communities (Companies) Regulations 1973 bringing into effect Section 3 of the European Communities Act 1972 (No 27 of 1972)
Moniteur Belge - Belgisch Staatsblad del 23.6.1973. p. 7681

Handelsmin., n. 119-1972. p. 1755

Journal Officiel de la République Française, n. 69193 del 28.12.1969

Bundesgezetzblatı, 1969, parte 1, p. 1146

Greek Official Jurnal, FIEK, 191 A del 28.11.1986

Greek Official Journal, FIEK, 197A del 10.12.1986 
ITALIA Decreto del Presidente della Repubblica del 29 dicembre 1969, n. 1127

LUSSEMBURGO Loi du 23 novembre 1972 portant adaptation de la loi du 10 août 1915 concernant le régime des sociétés commerciales telle qu'elle a été modifiée dans la suite à la directive $n$. 68/151 du Conseil des Communautés Européennes du 9 mars 1968

PAESI BASSI Wet van 29 april 1971. houdende aanpassing van de Nederlandse wetgeving aan de eerste richtlijn van de Raad van de Europese Gemeenschappen van 9 maart 1969 inzake het vennootschapsrecht

Portogallo Codigo das Sociedades Commerciais.

Decreto-Lei n. 262/86 de 2 de setembro.

Codigo do Registo Comercial.

Decreto-Lei n. 403/86 de 3 de Dezembro

REINO Unito European Communities Act 1972

SPAGNA

Ley 19/1989 de 25 julio 1989. de reforma parcial y adaption de la legislacion mercantil a las Directivas de la C.E.E. en materia de Sociedades
Gazzetta Ufficiale della Repubblica Italiana, n. 35 del 10.2.1970, p. 782

Mémorial, A-72 del 13.12,1972, pp. 1586-1594

Staatsblad van bet Koninkrijk der Nederlanden, 285. 1971
Boletin Oficial del Estado, do. B.O.E. del 27.7.1989

Seconda direttiva del Consiglio delle Comunità Europee del 13 dicembre 1976, n. $77 / 91$ intesa a coordinare, per renderle equivalenti, le garanzie che sono richieste, negli Stati membri, alle società di cui all'art. 58, secondo comma, del trattato per tutelare gli interessi dei soci e dei terzi per quanto riguarda la costituzione della società per azioni, nonché la salvaguardia e le modificazioni del capitale sociale della stessa (in G.U.C.E. n. L 26 del 31.1.1977, p. 1 s.)

Belgio Loi modifiant les lois sur les sociétés commerciales coordonnées le 30 novembre 1935 du 5.12.1984

Loi programme du 30.12 .88
Moniteur Belge - Belgisch Staatsblad, del 12.12.1984

Moniter Belge du 5.1.89. p. 161 
DANIMARCA Lov om aendring af lov om aktieselskaber Law no. 282 of 9 june 1982

Lov n. 1060 of 23.12 .1992

FRANCIA

GERMANIA

GRECIA

IRI.ANDA

ITALIA

LUSSEMBURGO

PAEsi BAssi

Portogallo

REGNO UNITO

SPAGNA
Loi no. 81-1162 du 30 décembre 1981 relative à la mise en hamonie du Droit des Sociétés commerciales avec la directive adoptée par le Conseil des Communautés Européennes le 13 décembre 1976

Gesetz zur Durchfuihrung der Zweiten Richtlinie des Rates der Europäischen Gemeinschaften zur Koordinierung des Gesellschaftsrechts. Vom 13. Dezember 1978

Presidential Decree n. 409/86 revising Law n. $2190 / 1920$ on public companies limited by shares

Loi n. 1882/90

Companies (Amendment) Act 1983 (No. 13 of 1983)

5 June 1983)

Decreto del Presidente della Repubblica del 10 febbraio 1986 , n. 30

Loi du 24 avril 1983 portant modification de la loi modifiés du 10 août 1915 concemant les société commerciales

Wet van 15 mei 1981 tot aanpassing van de wetgeving aan de tweede richtlijn van de Raad van de Europese Gemeenschappen inzake het vennootschapsrecht

Codigo das Sociedades Commerciais.

Decreto-Lei n. 262/86 de 2 de Setembro

Companies Act 1980. (Now revised and replaced by the Companies Act 1985).

Ley 19/1989 de 25 de julio 1989, de reforma parcial y adaption de la legislacion mercantil a las Directivas de la C.E.E. en materia de Sociedades
Lovtidende A, 1982. Haefie 46. p. 649

Journal Officiel de la République Française, del 31.12.1981.p. 3593

Bundesgezetzblatı. 1978, parte 1. p. 1959

Greek Official Journal, FEK, 191 A del 28.11.1986

Greek Official Journal. FEK. t3 del 23.3.1990

Gazetta Ufficiale, del 18.2.1986, n. 40

Mémorial A-35 del 16.5.1983. p. 864

Staatsblad van bet Kominkrijk der Nederlanden, 1981, 332

B.O.E., del 25.7.1989 
Direttiva del Consiglio del 23 novembre 1992, n. 92/101, che modifica la direttiva $77 / 91$ per quanto riguarda la costituzione della società per azioni, nonchè la salvaguardia e le modificazioni del capitale sociale della stessa (in G.U.C.E., n. L 347, del 28.11.92, p. 64 s.)

BELGIO

Arrêté Royale du 31.12.92

DANIMARCA

Lovbkg n. 616 af 19.7 .93

Lovbkg n. 662 af 12.8 .93

Bekendtgoerelse n. 533 af 13.7 .90

FRANCIA

ITALIA

PAesi BAssi

REGNO UNITO
Loi n. 66/537 du 24.7.66

Decret n. 67/236 du 23.3.67

Decreto legislativo dei 2.5.1994, n. 315
Moniteur Belge du 3.2.93, p. 2075

Gazzetta Ufficiale della Repubblica Italiana n. 121, del 26.5.94, p. 4

Statutory Instruments
$1932 / 1986$
Statutory Instruments
$1990 / 1504$ (N I 10)

1990/1504 (N I 10)

Terza direttiva del Consiglio del 9 ottobre 1978, n. 78/855, basata sull'art. 54, paragrafo 3, lettera g), del trattato e relativa alle fusioni delle società per azioni (in G.U.C.E. n. L 295, del 20.10.1978, p. 36 s.)

BELGIO

Loi n. 93-1774 du 29.6.93

DANIMARCA

FRANCIA
Art. 2 van het burgerliyk wetboek

The Companies Act 1985

The Companies (n. 1)

Order 1986

The Companies Act 1989

The Companies (n. 2 n. I)

Order 1990 
GERMANIA

\section{GRECIA}

IRLANDA

ITALIA

LUSSEMBURGO

PAESI BASSI

PortoGallo

REGNO UNITO

SPAgna
Gesetz zur Durchführung der Dritten Richtlinie des Rates der Europäischen Gemeinschaften zur Koordinierung des Gesellschaftsrechts.

Vom 25. Oktober 1982

Décree Presidentiel n. 498/87 portant modification de la loi n. 2190/1920

Statutory Instrument n. 137 of 1987

Decreto legislativo del 16.1.1991, n. 22
Bundesgezetzblatt, n. 40, parte 1, 1982, p. 1425

Journal Officiel, 236 del 31.12.1987

Iris Oifigiuil (Official Gazette), del 29.5.1987, p. 829

Gazzetta officiale della Repubblica Italiana, n. 19. del 23.1.1991

Mémorial, A-77, del 15.9.1987, p. 1972

Staatsblad, n. 59, 1983
Loi du 7 septembre 1987 modifiant la loi du 10 août 1915 concernant les société commerciales

Wet van 19 januari 1983 tot regeling van de fusie van naamlose en besloten venootschappen

Codigo das Sociedades Commerciais.

Decreto-Lei n. 262/86 de 2 Setembro

The Companies (Mergers and Divisions) Regulations 1987

Ley 19/1989 de 25 de julio 1989, de reforma parcial y adaptacion de la legislacion mercantil a las Directivas de la C.E.E. en materia de Sociedades
Statutory Instrument, n. 1991, del 1987

B.O.E., del 27.7.1989

Quarta direttiva del Consiglio del 25 luglio 1978, n. 78/660, basata sull'articolo 54, paragrafo 3 , lettera $\mathrm{g}$ ), del trattato e relativa ai conti annuali di taluni tipi di società (in G.U.C.E. n. L 222 del 14.8.1978, p. 11 s.)

Loi modifiant la loi du 17 juillet 1975 relative à la comptabilité et aux comptes annueles des entreprises - ler juillet 1983

Loi modifiant les lois, sur les sociétés commerciales, coordinnées le 30 novembre 1935 du 5 décembre 1984
Moniteur Belge - Belgisch Staatsblad del 8.7.1983

Moniteur Belge - Belgisch Staatsblad del 12.12.198t 
Arrêté royal portant exécution de la loi du 17 juillet 1975 relative à la comptabilité et aux comptes annuels des entreprises

Arrêté royal modifiant la loi du 17 juillet 1975 relatif à la comptabilité et aux comptes annuels des entreprises du janvier 1986

Arrété royal modifiant l'arrêté royal du 8 octobre 1976 relatif aux comptes annuels des entreprises - errata. 12 septembre 1983

Arrêté royal portant exécution de la loi du 17 juillet 1975 relative à la comptabilité et aux comptes annuels des entreprises Erratum. 12 septembre 1983

Arrêté royal modifiant l'arrêté royal du 8 octobre 1976 relatif aux comptes annuels des entreprises - errata. 12 septembre 1983

Arrêté royal portant execution loi du 17.7.75 - erratum du 12.9.83

Arrêté royal modifiant l'arrêté royal du 8.10 .76 - errata du 12.9 .83

DANIMARCA

Lov om visse selskabers aflaeggel-se af arsregnskab m.v.

Law 284 of 10 June 1981.

Lov om aendring af lov om aktieselskaber og lov om anpartsselskaber.

Law 285 of 10 June 1981

Bekendtgoerelse om regnskabsaflaeggelse af 17.12 .90

Lov n. 306 af 16.5.90, flere loue og bekendtgoerelse om regnskabsaflaeggelse of 17.12 .90

Lov bekendtgoerelse n. 22 af 9.1.1991

FRANCIA

Loi n. 83-353 du 30 avril 1983 relative à la mise en harmonie des obligations comptables des commerçants et de certaines sociétes avec la 4éme directive adoptée par le Conseil des Communautés Européennes le 25 juillet 1978
Moniteur Belge - Belgisch Staatsblad del 28.9.1983

Moniteur Belge - Belgisch Staatsblad del 28.1.1986

Moniteur Belge - Belgisch Staatsblad del 15.10.1983

Moniteur Belge .... Belgisch Staatsblad del 3.12.1983

Moniteur Belge - Belgisch Staatsblad del 28.9.1983

Moniteur Belge du 3.12.1983

Moniteur Belge du 3.12.1983

Industrimin

J. n. $101-2-80$ 
Décret n. 83-1020 du 29 novembre 1983 pris en application de la loi n. 83- $353 \mathrm{du}$ 30 avril 1983 et relatif aux obligations comptables des commerçants et de certaines sociétés.

Arrêté du 27 avril 1982 Approbation du plan comptable général révisé

GERMANIA

IRLANDIA

ITALIA

LUSSEMBURGO

PAESI BASSI

Portogallo

REGNO UNITO

SPAGNA
Bilanzrichtliniengesetz vom 19 Dezember 1985 S. 2355

Companies (Amendment) Act 1986

Decreto legislativo del 9 aprile 1991, n. 127

Loi du 4 mai 1984 portant modification de la loi du 10 août 1915 concernant les sociétés commerciales

Wet van 7 december 1983 houdende aanpassing van de wetgeving aan de vierde richtlijn van de Raad van de Europese Gemeenschappen inzake het venootschapsrecht

Besluit van 22 december 1983 houdende regels over de inhoud, de grenzen en de wijze van toepassing in de jaarrekening van waardering van activa tegen actuele waarde (Besluit waardering activa)

Besluit van 23 december 1983 tot vaststelling van modelschema's voor de inrichting van jaarrekeningen (Besluit modellen jaarrekening)

Decreto Lei n. 410/89, 21.11.89
Companies Act 1985 (Schedule 4)
Journal Officiel de la
République 1.12.1983, p. 3461

Journal Officiel de la République Française, n. 43/55 del 7.5.1982

Bundesgesetzblautt, 1985, parte 1, del 24.12.1985

Gazetta Ufficiale della Repubblica Italiana, Supplemento Ordinario, n. 90 del 17.4.1991, p. 5

Mémorial, A-40, del 10.5.1984

Staatsblad van bet Kominkrijk der Nederlanden 663

Staatsblad van bet Kominkrijk der Nederlanden, 665

Staatsblad van bet Kominkrijk der Nederlanden 666

Diario de Repubblica, I, n. 268 del 21.11.1989 p. 5112 (2)

Ley 19/1989 de 25 de julio 1989 de B.O.E., del 27.7.1989 reforma parcial y adaptacion de la legislacion mercantil a las Directivas de la C.E.E. en materia de Sociedades 
Direttiva del Consiglio dell' 8 novembre 1990, n. 90/604, che modifica la direttiva $78 / 660$ sui conti annuali e la direttiva $83 / 349$ sui conti consolidati per quanto riguarda le deroghe a favore delle piccole e medie società, nonché la pubblicazione dei conti in ecu (in G.U.C.E. n. L 317, del 16.11.90, p. 57 s.)

BELGIO

DANIMARCA

FRANCIA

GERMANIA

IRLANDA

ITALIA

\section{LUSSEMBURGO}

PAESI BASSI

Portogallo

REGNO UNITO
Arrêté Royal du 30.12.91

Lov n. 289 af 8.5.91

Lov n. 1059 af 23.12 .92 om aendring af lov om visse selskabers aflaeggelse af arsregnskab

Decret n. 94/663 du 2.8.94

Gesetz zur aenderung des D-markbilanzgesetzes und anderer handelsrechtlicher bestimmungen vom 25.7.94

E.C. (accounts) Regulations 1993

Decreto legislativo del 30.12.1992, n. 526
Moniteur Belge 31.12.91

Journal Officiel du 4.8.94, p. 11339

Bundesgesetzblatt (teile I, II, III) n. 47 von 29.7 .94 s.168?

S.I. n. 396 of 1993

Gazzetta Ufficiale della Repubblica Italiana, Supplemento Ordinario n. 7 del 11.1.93, p. 5

Loi du 29.7.93

Besluit van 10.6.93

Staatsblad n. 303, 1993

Diario da Republica I Serie n. 201, 2.9.86 p. 2340

Statutory Instruments n. 2452 of 15.10 .92

small and medium sized enterprises and Regulations 1992 of 15.10 .92

The Companies Act 1986 (Accounts of small and medium sized enterprises and publication of accounts in ecus) Statutory Instruments n. 503 
Direttiva del Consiglio dell' 8 novembre 1990, n. 90/605 che modifica le direttive $78 / 660$ e $83 / 349$ relative rispettivamente ai conti annuali e ai conti consolidati por quanto riguarda il loro campo di applicazione (in G.U.C.E. n. L 317 del 16.11 .90 , p. 60 s.)

$\begin{array}{ll}\text { BELGIO } & \text { Arrêté Royal du } 30.12 .91 \\ \text { DANIMARCA } & \begin{array}{l}\text { Lov n. } 1059 \text { af } 23.12 .92 \text { om aendring af lov } \\ \text { om visse selskabers aflaeggelse af } \\ \text { arsregnskab }\end{array}\end{array}$

Moniteur Belge du 31.12.91

FRANCIA

Decret n. $94 / 663$ du 2.8 .94

Journal Officiel du 4.8.94, p. 11339

GRECIA Loi n. 3190 de 1955

Decret Presidentiel n. 419 de 1986

Decret Presidentiel n. 325 du 6.10.94

FEK A. n. 174 du 24.10.94, p. 2104

Decret Presidentiel n. 326 du 6.10.94

FEK A. n. 174 du 24.10.94, p. 2104

IRLANDA E.C. (Accounts) Regulations 1993

Statutory Instruments n. 396 of 1993

LUSSEMBURGO Loi du 2.12.93

PAESI BASSI Besluit van 10.3.93

Staatsblad n. 303

REGNO UNITO The partnerships and unlimited companies (Accounts) Regulations 1993 of 20.7.1993

Sesta direttiva del Consiglio del 17 dicembre 1982, n. 82/891, basata sull'articoio 54, paragrafo 3 , lettera $\mathrm{g}$ ) del trattato e relativa alle scissioni delle società per azioni (in G.U.C.E. n. L 378 del 31.12.1982, p. 47 s.)

BELGIO Loi n. 93-1774 du 29.6.1993

Monitear Belge du 21.7. 1993, p. 17176

DANIMARCA Lov n. 1060 af 23.12.1992

FRANCIA Loi n. 88-17 du 5 janvier 1988 relative aux fusions et scissions de Sociétés commerciales, adoptée le 21.12.1987.

Journal Officiel, del 6.6.1998, p. 227 
GERMANIA Gesetz ueber die spaltung der von der Bundesgesetzblatt (teile I, II, treuhandanstalt verwalteten unternehmen III) $1,1,1991$, p. 854 del 5.4 .91

GRECIA Décret Présidentiel n. $498 / 87$ portant Journal OfficielI, del modification de la loi $2190 / 1920 \quad 31.12 .1987$

IRLANDA Statutory Instrument n. 137 of 1987 Iris (Officiel Gazette), del

I'TALIA Decreto legislativo 16.1.1991, n. 22 29.5.1987, p. 829

Gazzetta Ufficiale della Repubblica Italiana, n. 23, del 23.1.1991

LUSSEMBURGO Loi du 7 septembre 1988 modifiant la loi Mémorial, A-77 del 15.9.1987, du 10 août 1915 concernant les sociétés commerciales p. 1792

Portogallo Codigo das Sociedades Commerciais.

Decreto-Lei n. 262/86 de 2 de Setembro

REgno Unito The Companies (Merger and Divisions).

Statutory Instrument, n. 1991 Regulations 1987 del 1987

Spagna Ley 19/1989 de 25 de julio 1989, de reforma parcial y adaptacion de la legislacion mercantil a las Directivas de la C.E.E. en materia de Sociedades

Settima direttiva del Consiglio del 13 giugno 1983, n. 83/349, basata sull'art. 54, paragrafo 3 , lettera g), del trattato e relativa ai conti consolidati (in G.U.C.E. n. L 193 del 18.7.1983, p. 1 s.)

BELGIO

Arrêté Royal du 6.3. 1990

Arrêté Royal du 6.3.1990

Arrêté Royal du 6.3.1990

DINIMARCA Lov om andring af lov omviesse

Lov n. 308 af 16.5 .90

Bekendtgoerelse af 13.6 .90
Moniteur Belge, du 27.3.1990, p. 5675

Moniteur Belge, du 27.3.1990, p. 5718

Moniteur Belge, du 27.3.1990, p. 5719

Egnskab M.V Lovforslag, n. 130 af 17.1 .1990

$L M j$. n. 89-15.742 af 16.5.1990 $L M j$. n. 89-73.625 
FRANCIA

GERMANIA

GRECIA

IRLANDIA

ITALIA

LUSSEMIBURGO

PAesi Bass!

PORTOGALLO
Loi n. $85-11$ du 3 janvier 1985 relative aux comptes consolidés de certaines sociétés commerciales et entreprises publiques

Décret 86-221 du 17 février 1986

Arrêté Ministeriel du 1.2.1991

Bilanzrichtliniengesetz vom 19 Dezember 1985 , s. 2355

Gesetz zur aenderung des D-markbilanzgesetzes und anderer handelsrechtlicher bestimmungen vom 27.7.1994

Presidential Decree n. 409/86 revising law n. $2190 / 1920$ on public companies limited by shares (partial implementation).

Presidential Decree n. 419/86 revising Law n. 2190/1920 on "private limited companies" and "sociétés en commandite par actions".

European Communities (Companies: group accounts)

Regulations S.I., n. 201 of 1992

Decreto legisltaivo del 9.4.1991, n. 127
Journal Officiel de la République Française, del 4.1.1985, p. 101

Journal Officiel de la République Française, del 19.2.1986

Journal Officiel de la République Française, dell 22.2.1991, p. 2669

Bundesgesetzblatt, 1985, parte 1, del 24.12.1985, p.2355

Bundesgesetzblatt, (teile I,II, III), n. 47 vom 29.7.1994, p. 1682

Greek Official Journal, FEK, 191 A del 8.11.1986

Greek Official Journal, FEK, 197 A del 10.12.1986

Gazetta Ufficiale della Repubblica Italiana, n. 27 del 17.4.1991

Loi du 11 juillet 1988 relative à l'établissement des comptes consolidés

Wet van 10 november 1988, houdende bijzondere bepalingen voor de geconsolideerde jaarrehening

Decreto-Lei n. 238/91, 2.7.1991

Diario de Repubblica, I, Aviso n. 149, del 2.7.1991, p.3364

Decreto-Lei n. 36/92, 28.3.1992 Diario de Repubblica, 28.3.1992, p. 1482

\section{REgno Unito Statute Companies Act 1989, Chapte 40}


SPAGNA

Ley 19/1989 de 25 julio 1989 de reforma

B.O.E., del 27.7 .1989 parcial y adaptacion de la legislacion mercantil a las Directivas de la C.E.E. en materia de Sociedades

Ottava direttiva del Consiglio del 10 aprile 1984, n. 84/253, basata sull'art. 54, paragrafo 3 , lettera g), del trattato e relativa all'abilitazione delle persone incaricate del controllo di legge dei documenti contabili (in G.U.C.E. n. L 126 , del 12.5 .1984 , p. 20 s.)

BELGiO Loi relative à la réforme du révisorat d'entreprises du 21 février 1985

Moniteur Belge - Belgisch Staatsblad, 28.2.1985

Arrêté Royal du 17.3.

Moniteur Belge du, 27.3.1986, p. 4079

1986Arrêté Royal du 7.4.1986

Moniteur Belge du 11.4.1986

DANIMARCA Bekendtgoerelse om eksamen for statsautoriserede revisorer, n. 229 del 5.5.1986

Lov om aendring af lov om visse selskabers aflaeggelse af arsregnskab m.v., lov om aktieselskaber, lov om anpartsselskaber, lov om erhvervsdrivende fonde, lov om banker og sparekasser m.v., lov om statsautoriserede revisorer og lov om registrerede revisorer, n. 815 del 21.12.1988

Bekendtgoerelse af lov om statsautoriserede revisorer, n. 56 del 19.1.1989

Bekendtgoerelse n. 209 af 5.4.1991

Lavtinde A af 5.5.86

Lavtinde A af 21.12.88

Lavtinde A 1989 af 19.1.1989

Lavtinde A 1991 af 5.4.1991, p. 817

Bekendtgoerelse af 18.12.1991

Lov n. 427 af 1.6.1994

FRANCIA Loi du 24 juillet 1986 sur les sociétés commerciales.

Décret 69-810 du 12 août 1969 modifié.

Décret 81-536 du 12 mai 1981.

Décret 88-81 du janvier 1988.

Décret 88-60 du 22 janvier 1988 
GERMANIA

GRECIA

IRLANDA

ITALIA

LUSSEMBURGO

PAESI BASSI

Portogallo

REINO UNITO

SPAGNA
Bilanzrichtliniengesetz vom 19 Dezember 1985 S. 2355

Presidential Decree n. 15/89 of 3 January, 1989

The Company Act, 1990 (n. 33 of 1990)

(Part X Sec. 187/201)

The Company Act, 1990 (Auditors) Regulations

Commencement Order

Decreto legislativo del 27 gennaio 1992, n. 88

Loi du 28 juin 1984 portant organisation de la profession de réviseur d'entreprises Grand-Ducal Regulation du 16 août 1984, p. 1346

Wet van 6 augustus 1993 tot wijziging van de Wet op de Registeraccountants en de Wet op de Accountants-Administrativeconsulenten

Codigo das Sociedades Commerciais. Art. 446 et seq. D.R.I. n. 201 du 2.9.1986. Decreto-Lei No. 519-L2/79 de 29.12.1979

S. Companies Act, 1989, Chapter 40

Ley 19/1988 de 12 de julio de Auditoria de Cuentas
Bundesgesetzblautt, 1985, parte 1, del 24.12.1985, p. 2355

Greek Official Journal, FEK, n. 5 del 5.1.1989, vol A

Statutory Instruments, n. 259 of 1992

Statutory Instruments, n. 258 of 1992

Gazzetta Ufficiale della
Republica Italiana,
$\begin{aligned} & \text { Supplemento ordinário, n. } 37, \\ & \text { del 14.2.1992 }\end{aligned}$

Mémorial, A-81 del 23.8.1984

Staatsblad, n. 465, 1993

B.O.E., 15.7 .1989

Undicesima direttiva del Consiglio del 21 dicembre 1989, n. 89/666, relativa alla pubblicità delle succursali create in uno Stato membro da taluni tipi di società soggette al diritto di un altro Stato (in G.U.C.E. n. L 395, del 30.12.1989, p. $36 \mathrm{s.})$

\section{BELGIO}

DANIMARCA

Arrêté Royal du 30.12.91

Lov n. 289 af 8.5.91, s. 1080

Lov n. 127 af 26.2.92

FRANCIA
Décret n. 92/521 du 16.6.92
Moniteur Belge du 31.12.91

Lovtinde A 1992 Haefte 22 
GERMANIA Gesetz vom 22.7.93

GRECIA Décret presidentiel n. 360/93

IRLANDIA E.C. (Accounts) Regulations 1993

I'TALIA

LUSSEMBURGO Loi du 27.11.92

PAESI BASSI Produktschap voor vee en vlees van 29.10.92 tot wijziging van handelsregisterw

Besluit van 24.12.92 tot wijziging van het Staatsblad 1992, n. 667 handelsregisterw

Produktschap voor vee en vlees van 3.7.93, haudende wijziging van 3.7 .93 , haudende wijziging van handelsregisterw

Mededeling van minister

Portogallo Decreto-lei n. 262/86, 2.9.86

Decreto-lei n. 225/92, 21.10.92

Regno Unito The Company Act 1985 (disclosure of branches and bank accounts) Regulations 1992

The Oversea companies and credit and financial institutions (branch disclosure) Regulations 1992

Companies (1986 order) (disclosure of branches accounts) Regulations (Northern Ireland) 1993

E.C. (Branch disclosures) Regulations 1993

SPAGNA
Real decreto n. 1597/89, 29.12.89
Bundesgesetzblatt (teile I, II, III) I n. 39 s. 1282 vom 29.7 .93

FEK A n. 154 du 13.9 .93

S.I. n. 396 of 1993

Gazzetta Ufficiale della Repubblica Italiana n. 306, del 31.12 .92

Memorial A n. 95 du 15.12.92

Staatsblad 1992, n. 595

Staatsblad 1993, n. 389

Staatscourant van 3.1 .93 , n. 8 Diario de Republica, I Serie n. 201, 2.9.86, p. 2293

Statutory Instruments n. 3178 of 13.12 .92

Statutory Instruments n. 3179 of 13.12 .92

Statutory Rules of Nortbern Ireland n. 199 of 14.4 .1993

Statutory Instruments n. 395 of 1993

Bolitin Oficial del Estado n. 313, 30.1.89, p. 2762 
Dodicesima direttiva del Consiglio del 21 dicembre 1989, n. 89/667, in materia di diritto delle società relativa alle società a responsabilità limitata con un unico socio (in G.U.C.E., n. L 395, del 30.12.1989, p. 40 s.)

$\begin{array}{ll}\text { BELGIO } & \text { Loi du } 14.7 .87 \\ \text { DANIMARCA } & \text { Lov n. } 289 \text { af } 8.5 .91, \text { p. } 1080 \\ \text { FRANCIA } & \text { Loi n. } 85 / 697 \text { du } 11.7 .85\end{array}$

Décret n. 88/909 du 30.7 .88

GERMANIA Gesetz zur Durchführung der Zwölften Richtlimie des Rates der Europäischen Gemeinschaften auf dem Gebiet des Gesellschaftsrechts betreffend Gesellschaften mit beschränkter Haftung mit einem einzigen Gesellschafter vom 18.12.1991

GRECIA Décret Presidentiel n. 279/1993

IRLANDA European Communities (Single-Member private limited companies) Regulations 1994

European Communities (Single-Member private limited companies) (Forms) Regulations 1994

ITALIA Decreto legislativo n. 88 del 3.3.93

LUSSEMBURGO Loi du 28.12.92 modifiant loi du 10.8.15

PAESI BASSI Besluit van 24.1.92

Wet van 19.12.1991

PORTOGALLO Decreto-lei 248/86 25.8.86
Moniteur Belge du 30.7.87

Journal Officiel da 12.7.85, p. 7082

Journal Officiel da 4.8.88, p. 9869

Bundesgesezblautt Teil I, z.. 5702 A., n. 66, 1991 vom 24.12.91. Seire 2206

FEK A n. 122 du 27.7.93

Statutory Instruments n. 275 of 1994

Statutory Instruments n. 306 of 1994

Gazzetta Ufficiale della Repubblica Italiana, Supplemento Ordinario, n. 78, del 3.4 .93 , p. 5

Memorial A n. 106 da 30.12 .92 , p. 3141

Staatsblad 1992 n. 52

Staatsblad 1991 n. 710 
REgno UNITO Companies (Single-Member private limited companies) Regulations (Northern Ireland) 1992 of 21.9 .92

Companies (Single-Member private limited companies) Regulations 1992 of 14.7 .92
Statutory Rules of Nortbern Ireland n. 405 of 1992

Statutory Instruments n. 1699 , 1992

Roma, settembre de 1997. 